\title{
Cardiac Mechanical Parameter Calibration Based on the Unscented Transform
}

\author{
Stéphanie Marchesseau ${ }^{1, \star}$, Hervé Delingette ${ }^{1}$, Maxime Sermesant ${ }^{1}$, \\ Kawal Rhode ${ }^{2}$, Simon G. Duckett ${ }^{2}$, C. Aldo Rinaldi ${ }^{2}$, \\ Reza Razavi², and Nicholas Ayache ${ }^{1}$ \\ 1 Asclepios Research Project, INRIA Sophia Antipolis, France \\ 2 King's College London, Division of Imaging Sciences \& Biomedical Engineering, \\ St. Thomas' Hospital, London, UK
}

\begin{abstract}
Patient-specific cardiac modelling can help in understanding pathophysiology and predict therapy planning. However it requires to personalize the model geometry, kinematics, electrophysiology and mechanics. Calibration aims at providing global values (space invariant) of parameters before performing the personalization stage which involves solving an inverse problem to find regional values. We propose an automatic calibration method of the mechanical parameters of the BestelClément-Sorine (BCS) electromechanical model of the heart based on the Unscented Transform algorithm. A sensitivity analysis is performed that reveals which observations on the volume and pressure evolution are significant to characterize the global behaviour of the myocardium. We show that the calibration method gives satisfying results by optimizing up to 7 parameters of the BCS model in only one iteration. This method was evaluated on 7 volunteers and 2 heart failure patients, with a mean relative error from the real data of $11 \%$. This calibration enabled furthermore a preliminary study of the specific parameters to the studied pathologies.
\end{abstract}

\section{Introduction}

Patient-specific cardiac modelling can provide additional guidance to cardiologists in understanding pathophysiology and predict therapy planning. Several approaches for the past 20 years have been developed to describe and simulate the cardiac function, including cardiac mechanics and electrophysiology [4]. They differ in their choice of hyperelastic material, electrophysiological properties or electromechanical coupling. In this paper the Bestel-Clement-Sorine (BCS) model [1], further improved by [3] is used.

The simulation becomes patient-specific after several levels of personalization: geometrical, kinematic, electrophysiological and mechanical. Mechanical personalization consists in optimizing mechanical parameters of the model so that the

\footnotetext{
* Note: This work was partially funded by the European Community's euHeart project under grant agreement 224495 and by the ERC advanced Grant MedYMA.
} 
simulation behaves in accordance to patient-specific datasets (images and other signals).

This inverse problem has been tackled by different authors (for instance 12 , [7, [8] or [10]). However there is no guarantee that such algorithms will converge toward a relevant solution due to their dependence on an initial range of parameter values. The choice of the parameters to estimate and their initial calibration has therefore great impact for the personalization.

Our main contribution tackles this initialization issue: we propose a simple and efficient method to automatically calibrate the parameters from the ventricular volume or pressure evolution over the cardiac cycle. It has been applied successfully for the calibration of mechanical parameters from 7 healthy cases and has been tested in two heart failure cases. Our proposed method is based on the Unscented Transform algorithm and requires only one iteration with multiple simulations performed in parallel for calibrating up to 7 parameters selected from a sensitivity analysis. Moreover, a comparison between the estimated parameters for control and heart failure cases enabled a preliminary specificity study that aims at classifying the pathologies.

\section{The Bestel-Clement-Sorine Electromechanical Model of the Heart}

Our approach is based on the Bestel-Clement-Sorine (BCS) model [1] further improved by 3 . The model is composed of a passive isotropic visco-hyperelastic component that accounts for the elasticity and the friction in the cardiac extracellular matrix surrounding the fibres, described as a Mooney-Rivlin material. The strain energy for a Mooney-Rivlin material is given as: $W_{e}=c_{1}\left(\bar{I}_{1}-3\right)+$ $c_{2}\left(\bar{I}_{2}-3\right)+\frac{K}{2}(J-1)^{2}$, where $c_{1}, c_{2}$ are material parameters and $K$ is the Bulk modulus. The quantities $\bar{I}_{1}$ and $\bar{I}_{2}$ are the isochoric invariants of the Cauchydeformation tensor $\mathbf{C}$.

In parallel, the stress along the cardiac fibre is composed of an active part (contraction in the sarcomere) and a passive part corresponding to the elastic bound (titin) between sarcomeres and Z-discs, having stress $\sigma_{s}=E_{s} e_{s}$. The contractile component having stress tensor $\sigma_{c}$, driven by the control variable $u$, has a viscous part to account for the energy dissipated in the sarcomere due to friction. This gives $\sigma_{c}=\tau_{c}+\mu \dot{e_{c}}$. Fig. 1 shows a rheological representation of this model.

At the nanoscopic scale, the binding and unbinding process of the actin and myosin filaments in the sarcomere is described by Huxley's filament model [5]. Statistical mechanics allows to describe its behavior at the macroscopic scale, resulting in a differential equation that controls the active stress $\tau_{c}$ and the sarcomere stiffness $k_{c}$ :

$$
\left\{\begin{array}{l}
\dot{k_{c}}=-\left(|u|+\alpha\left|\dot{e_{c}}\right|\right) k_{c}+n_{0} k_{0}|u|_{+} \\
\dot{\tau_{c}}=-\left(|u|+\alpha\left|\dot{e_{c}}\right|\right) \tau_{c}+\dot{e_{c}} k_{c}+n_{0} \sigma_{0}|u|_{+}
\end{array}\right.
$$

where $\alpha$ is a constant related to the cross-bridge release due to a high contraction rate, $k_{0}$ and $\sigma_{0}$ are respectively the maximum stiffness and contraction. $n_{0}$ is 

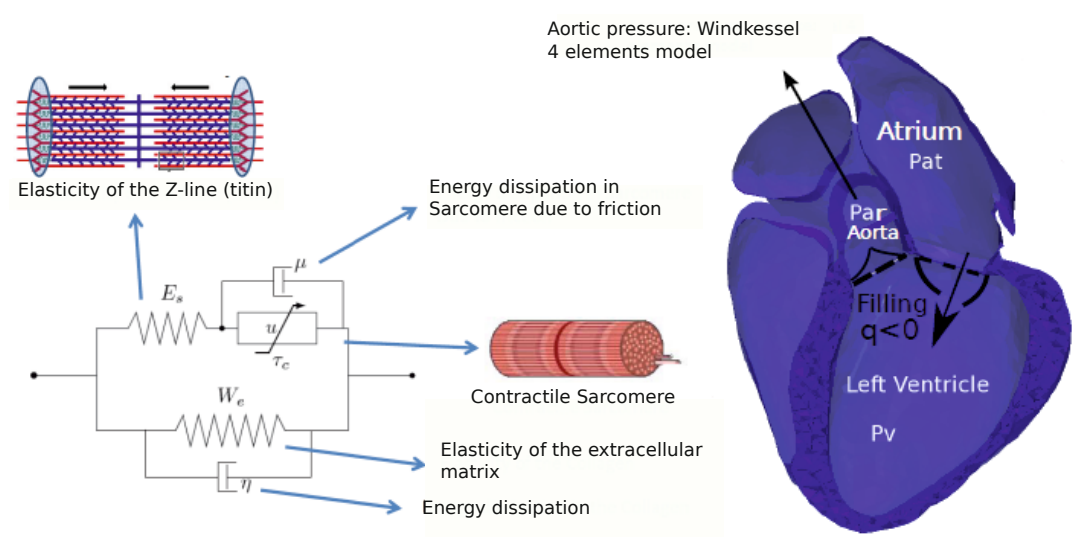

Fig. 1. Full electromechanical and circulation model. (Left) $W_{e}$ is the strain energy of the extracellular matrix considered here as an isotropic material, associated with a dissipative term $\eta . u$ is a control variable which is driven by changes in transmembrane potential. It controls the contraction stress $\tau_{c} . \mu$ deals with the friction in the sarcomere while $E_{s}$ is a linear spring to enforce elasticity of the Z-discs (titin). (Right) Circulation model in the filling phase for the left ventricle.

a reduction factor that allows to take into account the Starling effect by which the maximum contraction depends on the fibre strain $e_{c}$. The control variable $u$ is derived from the electrical activation model and is a function of the free calcium concentration only. It is modeled using electrophysiological inputs such as depolarization times $\left(T_{d}\right)$ and action potential durations $(A P D)$ and depends on two parameters: $k_{A T P}$ the rate of the myosin ATPase activity controlling the contraction rate and $k_{R S}$ the rate of sarcoplasmic reticulum calcium re-uptake controlling the relaxation rate.

The ventricles are filled with blood coming from the atria and ejected through the arteries. A basic circulation model is represented in Fig. 1, A valve model explained in 3 gives relationships between the outward flow and the various pressures (ventricular, arteria and atria). The arteria pressure is modeled using a four-element Windkessel model [9], that depends on four parameters: the peripheral resistance $R_{p}$, the characteristic time $\tau$, the characteristic resistance $Z_{c}$ and the total arteria inertance $L$.

\section{Unscented Transform-Based Parameter Calibration}

To calibrate the model, we use the algorithm derived from the Unscented Transform [6]. We chose the ventricular volume curves as main observations to perform the calibration as they are important physiological indices and can be captured by few quantities: the minimum volume $V_{\min }$, the maximum and minimum of the flow $\left(q_{\max }\right.$ and $q_{\min }$ respectively). Moreover, if available, the maximum pressure 
$P_{\max }$, as well as the maximum and minimum of the derivative of the pressure $\left(d P / d t_{\max }\right.$ and $d P / d t_{\min }$ respectively) can be taken into account.

\subsection{Unscented Transform Algorithm}

The proposed algorithm finds a set of parameters that minimize the difference between the measured observation $\mathbf{Z}^{\text {obs }}$ and the predicted observation $\overline{\mathbf{Z}}$. It is explained as follows: Let $\mathbf{Z}$ be the vector of observations and $\mathbf{X}$ the parameter vector which has mean $\mathbf{X}^{0}$, covariance $\mathbf{C}_{X}$ and dimension $n$. We set the covariance as $\mathbf{C}_{X}=\operatorname{Cov}(\mathbf{X}, \mathbf{X})$ by estimating the minimal and the maximal value of each parameter with a trial and error approach on one volunteer. We compute observations $\mathbf{Z}_{i \epsilon}$ from the $2 n+1$ sets of parameters $\mathbf{X}_{i \epsilon}=\left[x_{1}, x_{2}, \ldots, x_{i}+\epsilon s_{i}, \ldots\right]$ around the mean value $\mathbf{X}^{0}$ where $\epsilon \in\{-1,0,1\}$ and $s_{i}$ is an uncertainty function of the covariance $s_{i}=\gamma{\sqrt{\mathbf{C}_{X}}}_{i}$, with $\gamma$ the scaling parameters. The mean observation is set as $\overline{\mathbf{Z}}=\sum_{i, \epsilon} \omega_{i \epsilon} \mathbf{Z}_{i \epsilon}$ with some weights $\omega_{i \epsilon}$ described by [11]. Finally we derive the covariance matrix as:

$$
\operatorname{Cov}(\mathbf{X}, \mathbf{Z})=\sum_{i \epsilon} \omega_{i \epsilon}\left(\mathbf{X}_{i \epsilon}-\mathbf{X}^{0}\right)\left(\mathbf{Z}_{i \epsilon}-\overline{\mathbf{Z}}\right)^{T}
$$

The new set of parameters $\mathbf{X}^{\text {new }}$ found to match the observations $\mathbf{Z}^{\text {obs }}$ is

$$
\left(\mathbf{X}^{n e w}-\mathbf{X}^{0}\right)=\operatorname{Cov}(\mathbf{X}, \mathbf{Z}) \operatorname{Cov}(\mathbf{Z}, \mathbf{Z})^{-1}\left(\mathbf{Z}^{o b s}-\overline{\mathbf{Z}}\right)
$$

where

$$
\operatorname{Cov}(\mathbf{Z}, \mathbf{Z})=\sum_{i \epsilon} \omega_{i \epsilon}\left(\mathbf{Z}_{i \epsilon}-\overline{\mathbf{Z}}\right)\left(\mathbf{Z}_{i \epsilon}-\overline{\mathbf{Z}}\right)^{T}
$$

This algorithm is very simple to implement and runs in one iteration to give $\mathbf{X}^{\text {new }}$. Another simulation is necessary to obtain the resulting observation $\mathbf{Z}^{\text {new }}$.

\subsection{Parameter Selection}

Fourteen parameters in total have to be estimated: $\left(\sigma_{0}, k_{r s}, k_{a t p}, k_{0}, \alpha, \mu, E_{s}\right)$ active parameters, $\left(K, c_{1}, c_{2}\right)$ passive parameters and $\left(R_{p}, \tau, Z_{c}, L\right)$ for the valve model. Since it is not reasonable to try to estimate all of them at once, we decide to fix some to a standard value and estimate others. A sensitivity analysis was performed in order to select the main parameters. We chose the following four parameters $\left(\sigma_{0}, \mu, K, R_{p}\right)$ that we might be able to estimate from a volume curve. This choice was confirmed by a Singular-Value-Decomposition (SVD) of the covariance matrix $\operatorname{Cov}(\mathbf{X}, \mathbf{Z})$ made from all fourteen parameters and the three observations $\left(V_{\min }, q_{\max }\right.$ and $\left.q_{\min }\right)$. When pressure curves are available, more parameters can be estimated. $k_{r s}, k_{a t p}$ as well as the stiffness parameter $c_{1}$ were chosen since they greatly influence the pressure slopes. Relevant curves are presented in Fig. 2. 


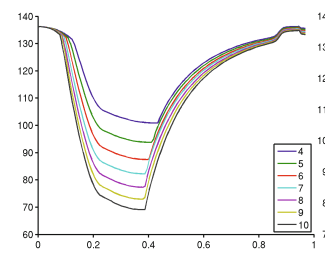

(a) $\sigma_{0}(\mathrm{MPa})$

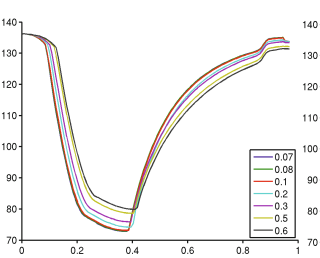

(b) $\mu$ (MPa.s)

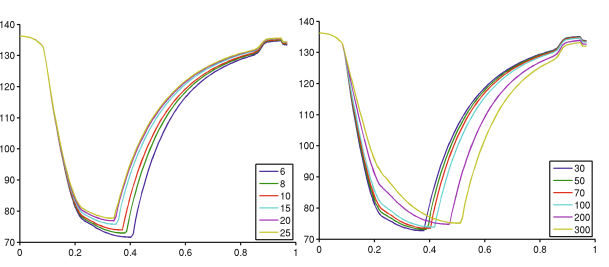

(c) $K(\mathrm{MPa})$

(d) $R_{p}\left(\mathrm{MPa} \cdot \mathrm{m}^{-3} \cdot \mathrm{s}\right)$

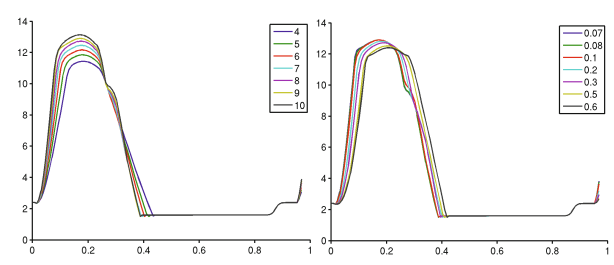

(e) $\sigma_{0}(\mathrm{MPa})$

(f) $\mu$ (MPa.s)

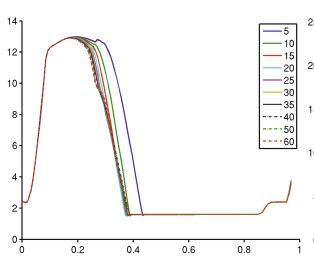

(g) $k_{r s}\left(\mathrm{~s}^{-1}\right)$

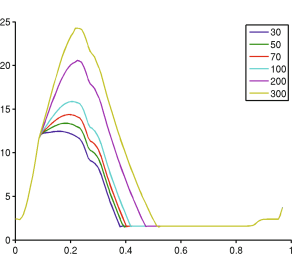

(h) $R_{p}\left(\mathrm{MPa}_{\mathrm{m}}^{-3} \cdot \mathrm{s}\right)$

Fig. 2. Observations over time: (Top) Volume in $\mathrm{mL}$, (Bottom) Pressure in $\mathrm{kPa}$

\section{Calibration Results on Healthy and Pathological Cases}

We demonstrate the application of the proposed method on cardiac MRI data on both volunteer and patient data acquired at the Division of Imaging Sciences \& Biomedical Engineering at King's College London, UK. Initial parameters $X^{0}$ and covariance matrix $\mathbf{C}_{X}$ are the same for all cases. Each case was calibrated in about 20 minutes that includes the time to run in parallel the $2 n+1$ simulations, the calibration time and the final simulation with the calibrated parameters.

\subsection{Volunteer Data: Calibration with Volume Curves}

The study was performed on seven healthy hearts provided by the STACOM challenge. The electrophysiological model was simulated with standard values and healthy onset (see Fig 3 Left). From the kinematic personalization, we registered all images on the end diastolic image. Then, image transformations were applied to the end-diastolic tetrahedral mesh to estimate the volume of the ventricles over time and then the observation vector $\mathbf{Z}^{\text {obs }}$. Fig. 3] shows the measured, reference and estimated volume curves on case 3 and errors between the real observations $\mathbf{Z}^{\text {obs }}$ and the simulated observations $\mathbf{Z}^{\text {new }}$ are given in Table 1 Moreover, the calibration provided a consistent and plausible range of global values for parameters that will be used in a specificity study (see Table 21).

\subsection{Pathological Data: Calibration with Volume and Pressures}

The proposed calibration approach was applied on two Left Bundle Branch Block (LBBB) 11 cases. Electrophysiological personalization (see Fig. 4left) was enabled

\footnotetext{
${ }^{1}$ LBBB cases are characterized by dyssynchronous electrophysiology.
} 
Table 1. Relative errors (in \%) between simulated results $\mathbf{Z}^{\text {new }}$ and real data $\mathbf{Z}^{\text {obs }}$ on the 7 healthy cases

\begin{tabular}{|c||c|c|c|c|c|c|c||c|}
\hline Volunteers & 1 & 2 & 3 & 4 & 5 & 6 & 7 & Mean \\
\hline \hline$V_{\min }$ & 0.35 & 3.51 & 0.83 & 0.79 & 1.09 & 1.38 & 1.31 & 1.32 \\
$q_{\max }$ & 3.06 & 20.99 & 8.57 & 21.37 & 11.5 & 12.1 & 5.36 & 11.85 \\
$q_{\min }$ & 0.31 & 4.12 & 27.41 & 6.48 & 27.58 & 16.92 & 5.81 & 12.66 \\
\hline
\end{tabular}

Table 2. Estimated parameters from the calibration

\begin{tabular}{|c||c|c|c|c|c|c|c||c|}
\hline Volunteers & 1 & 2 & 3 & 4 & 5 & 6 & 7 & Mean \\
\hline \hline$\sigma_{0}(\mathrm{MPa})$ & 6.49 & 4.42 & 4.92 & 5.46 & 5.51 & 8.75 & 5.32 & 5.8 \\
$\mu(\mathrm{MPa} . \mathrm{s})$ & 0.31 & 0.31 & 0.27 & 0.3 & 0.33 & 0.26 & 0.3 & 0.3 \\
$K(\mathrm{MPa})$ & 14.22 & 10.44 & 12.72 & 13.24 & 14.12 & 12.72 & 12.82 & 12.9 \\
$R_{p}\left({\mathrm{MPa} . \mathrm{m}^{-3}}^{-\mathrm{s}}\right)$ & 93.87 & 130.88 & 110.1 & 116.73 & 104.43 & 98.3 & 141.39 & 113.7 \\
\hline
\end{tabular}
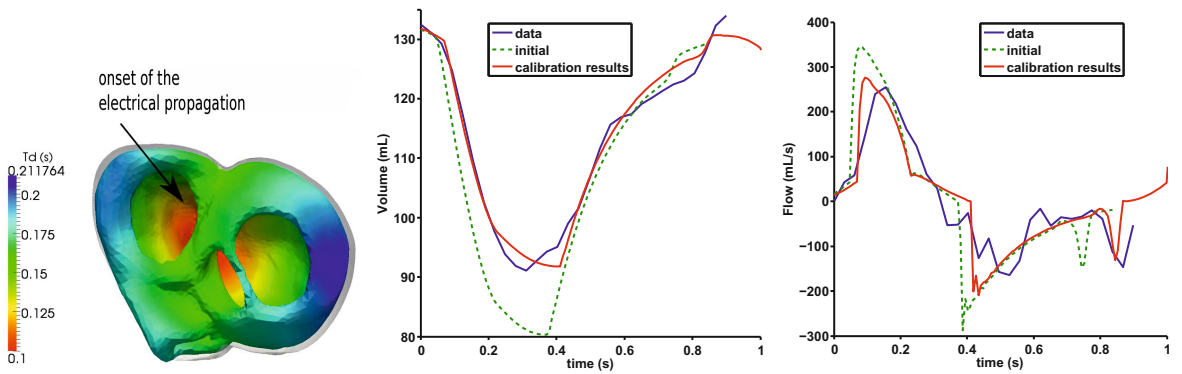

Fig. 3. (Left) Electrophysiological and geometrical input. (Right) Results of the calibration technique on real data for one healthy volunteer.

and pressure curves were available thanks to pressure sensors. Results on the two LBBB cases are given in Fig. 4 and the comparison with the images is given in Fig. 5. The mean relative error for both cases is about $16 \%$. We noticed a much higher passive stiffness $(K=19 \mathrm{MPa})$ for LBBB case 2 which also suffers from dilated cardiomyopathy, and a smaller contractility $\left(\sigma_{0}<4 \mathrm{MPa}\right)$ for both heart failure cases. These results are in agreement with medical knowledge (see [2] for instance). Therefore we can assess that a small contractility may be specific to LBBB cases as a high myocardial stiffness may be to dilated cardiomyopathy.

\subsection{Evaluation of Registration Error Influence}

We tried to evaluate the error in the registration technique to understand whether the model could actually match the data better than shown in Fig. 3. For this purpose, we created synthetic images from a real sequence, using the deformed meshes resulting from a simulation. We then registered this new sequence with the same registration technique as used for the kinematics personalization, and extracted the volume curves from the resulting registered meshes. The comparison between 

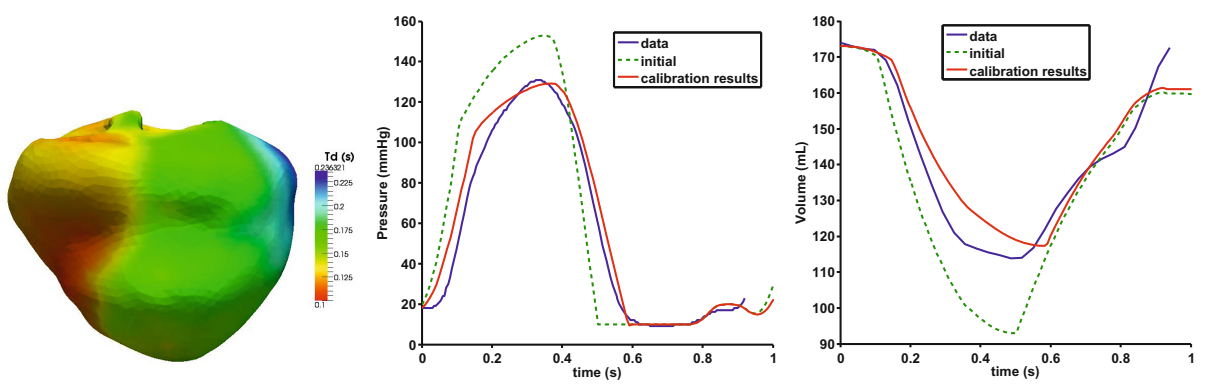

Fig. 4. (Left) Personalized electrophysiology. (Right) Results of the calibration technique on real data for $\mathrm{LBBB}$ case 1 .

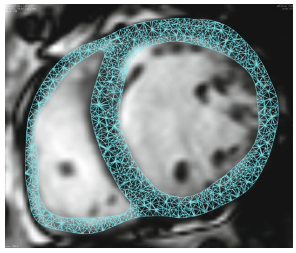

(a) $\mathrm{t}=0 \mathrm{~ms}$
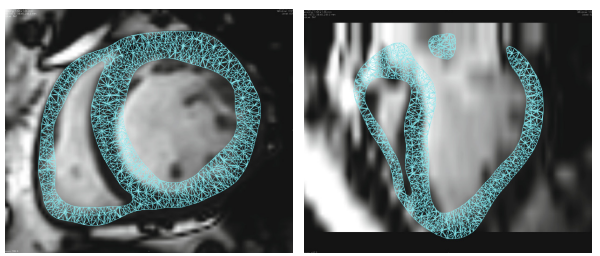

(b) $\mathrm{t}=410 \mathrm{~ms}$

Fig. 5. Simulated mesh for LBBB case 1

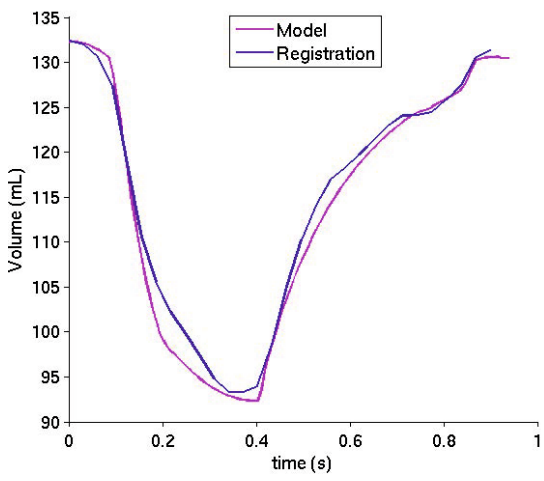

Fig. 6. Comparison between the volume variation computed from the simulation and the one estimated from registered images

the initial simulated volume curve and the one computed after registration gives a relative error of about $25 \%$ for both slopes (see Fig [6), which is of the same order of magnitude as the one after model calibration.

\section{Conclusion}

In this paper we proposed an innovative calibration method of an electromechanical cardiac model. The model depends on 14 parameters that act on the active, passive and constraint components. The calibration based on the Unscented Transform allowed us to give a fast initialization of 4 or 7 parameters, leaving the others fixed to standard values. The choice of these parameters was made based on a sensitivity analysis on the volume and pressure variation and 
confirmed by a Singular-Value-Decomposition analysis. Since the calibration requires only to run several simulations in parallel to estimate these parameters followed by one additional simulated cycle to verify the results, it can easily be used as a preprocessing step before the application of more sophisticated personalization algorithms. Moreover, the calibration performed on the 7 healthy volunteers and 2 heart failure cases allowed us to compare parameters for pathological cases versus healthy controls as a first step toward specificity analysis to classify various pathologies. Additional heart failure cases and observations quantities (global indices of the strain for instance) are required to further improve the calibration and validate this specificity study. Finally, the impact of the calibration on the personalization algorithm also needs to be investigated.

\section{References}

1. Bestel, J., Clément, F., Sorine, M.: A Biomechanical Model of Muscle Contraction. In: Niessen, W.J., Viergever, M.A. (eds.) MICCAI 2001. LNCS, vol. 2208, pp. 1159-1161. Springer, Heidelberg (2001)

2. Bortone, A.S., Hess, O.M., Chiddo, A., Gaglione, A., Locuratolo, N., Caruso, G., Rizzon, P.: Functional and structural abnormalities in patients with dilated cardiomyopathy. J. Am. Coll. Cardiol. 14, 613-623 (1989)

3. Chapelle, D., Le Tallec, P., Moireau, P., Sorine, M.: An energy-preserving muscle tissue model: formulation and compatible discretizations. Int. J. MultiScale Comput. Eng. (IJMCE) 10(2), 189-211 (2012)

4. Hunter, P., Nash, M., Sands, G.: Computational electromechanics of the heart. In: Computational Biology of the Heart, pp. 345-407 (1997)

5. Huxley, A.: Muscle structure and theories of contraction. Progress in Biophysics and Biophysical Chemistry 7, 255 (1957)

6. Julier, S., Uhlmann, J.: A new extension of the Kalman filter to nonlinear systems. In: Int. Symp. Aerospace/Defense Sensing, Simul. and Controls., vol. 3, p. 26. Citeseer (1997)

7. Liu, H., Shi, P.: Maximum a posteriori strategy for the simultaneous motion and material property estimation of the heart. IEEE Trans. Biomed. Eng. 56(2), 378389 (2009)

8. Moireau, P., Chapelle, D.: Reduced-order Unscented Kalman Filtering with application to parameter identification in large-dimensional systems. COCV 17, 380-405 (2011)

9. Stergiopulos, N., Westerhof, B., Westerhof, N.: Total arterial inertance as the fourth element of the windkessel model. Am. J. Phys (Heart and Circulatory Phys.) 276(1), H81 (1999)

10. Sundar, H., Davatzikos, C., Biros, G.: Biomechanically-Constrained 4D Estimation of Myocardial Motion. In: Yang, G.-Z., Hawkes, D., Rueckert, D., Noble, A., Taylor, C. (eds.) MICCAI 2009, Part II. LNCS, vol. 5762, pp. 257-265. Springer, Heidelberg (2009)

11. Wan, E., Van Der Merwe, R.: The unscented Kalman filter for nonlinear estimation. In: AS-SPCCS, pp. 153-158. IEEE (2000)

12. Xi, J., Lamata, P., Lee, J., Moireau, P., Chapelle, D., Smith, N.: Myocardial transversely isotropic material parameter estimation from in-silico measurements based on a reduced-order unscented Kalman filter. J. Mech. Behav. Biomed. Mater (JMBBM) 4, 1090-1102 (2011) 